\title{
LOS PROYECTOS DE LEY SOBRE SOFTWARE LIBRE EN COLOMBIA
}

\section{Alexander Pereira-García}

(D) https://orcid.org/0000-0001-5240-1365

(1) Corporación Universitaria Minuto de Dios (Uniminuto), Bogotá - Distrito Capital, Colombia. Email: alexander.pereira@uniminuto.edu.co

DOI: $10.1590 / 3510406 / 2020$

\section{Regular el Software Libre}

La regulación tecnológica constituye un asunto relevante para los países dado el rol central que cumplen las tecnologías de la información y la comunicación en el contexto social contemporáneo, particularmente en las dinámicas de la nueva economía digital (Cepal, 2013) y la sociedad del conocimiento (Scarso, 2008). La importancia de las tecnologías en la vida práctica se refleja materialmente en la presencia creciente de artefactos -electrónicos y digitales- en la cotidianidad (Winocur, 2007), también socialmente, como resultado de un discurso dominante que afirma la capacidad de la tecnología para dinamizar los procesos de cambio social, específicamente, el logro de la eficiencia, la competitividad y el desarrollo (Castells, 2002). Los vínculos entre imaginarios tecnológicos y sociales

Artigo recebido em: 02/05/2019

Aprovado em: 06/01/2020 se expresan en los modos de vida desarrollados en la contemporaneidad (Vera, 2013), como construcción de una realidad que se atraviesa por procesos de institucionalización (Castoriadis, 2013). En tal sentido, los imaginarios sobre las tecnologías adquieren legitimidad en los procesos de normalización estatal, siendo la regulación de las tecnologías un eje central de la política pública actual, cuyo fin último es el de hacerse con el control sobre sus formas de producción, uso e intercambio.

La presencia creciente de múltiples tecnologías en el contexto de las prácticas sociales y la vida cotidiana conduce a una densificación de las relaciones entre tecnologías y personas (Sabater, Martínez \& Santiago, 2017). De allí que se afirme que las tecnologías informacionales cumplen un papel estratégico en la estructuración de las relaciones sociales del mundo contemporáneo (Castells, 2002). Sin embargo, a pesar de la presencia generalizada de artefactos, técnicas y desarrollos tecnológicos diversos, los cuales 
se muestran como posibilidades -e incluso como condiciones- para el mejoramiento de la calidad de vida de los individuos y los grupos sociales (White, 1990), no existe una postura homogénea sobre tales relaciones entre tecnologías y sociedades (Nye, 2006). Por el contrario, diversos colectivos han asumido una postura crítica respecto a las hegemonías que, afirman, se construyen en torno a las tecnologías, las cuales pueden conducir a prácticas de control social (Franklin, 2013). Como consecuencia, diversos grupos se han organizado con el objetivo de promover la satisfacción de sus propias prerrogativas y, en el caso particular de las tecnologías informacionales, o TIC, como actores que promueven su democratización (Kellner, 2001), teniendo en cuenta las posibilidades de exclusión social y digital en algunas regiones, en relación con el acceso a objetos digitales y culturales (De Greiff, 2017). La garantía de acceso se entiende como un ejercicio que favorece la libertad y soberanía tecnológica, así como el disfrute de los derechos y beneficios que suponen las TIC (Andrade \& Campo-Redondo, 2012), en el marco de sociedades democráticas.

En este contexto, la regulación del software representa una dimensión particular de la regulación tecnológica, constituyendo el campo normativo de uno de los sectores más importantes para la economía global (Cepal, 2016). La creciente relevancia del software, con la popularización de las tecnologías computacionales, derivó en un asunto de interés público, una controversia sociotécnica relativa a las alternativas de producción, uso y distribución del software, la cual comprometió a diversos sectores sociales y se constituyó en una disputa de carácter político (Guesser, 2006). Hacia las décadas de 1980 y 1990 la discusión sobre las ventajas y conveniencia de diferentes modelos de software resultó en la alineación de individuos y grupos en torno a lo que consideraron propuestas adecuadas para la producción y distribución de esta tecnología (Gómez, 2003; Hess, 2005; Söderberg, 2008). Si bien el antagonismo entre los dos principales modelos de desarrollo y comercialización del software sugiere importantes diferencias técnicas, es en el contexto de la propiedad intelectual, o el licenciamiento del software, en donde se ubican las principales luchas y se enmarca inicialmente la discusión entre software privativo y software libre (De Laat, 2005).

Los dos tipos principales de licencia de software otorgan diversas prerrogativas a los actores involucrados: productores, empresarios y usuarios. La posibilidad de disposición del código fuente ${ }^{1}$ al usuario final implica una postura con dimensiones económicas y políticas. En el caso del software propietario o privativo, las licencias constituyen la base de las relaciones entre distribuidores y clientes, el código fuente es propiedad del productor mientras que el usuario tiene únicamente la posibilidad de usar las aplicaciones finales. Lo anterior se sostiene en la lógica de los incentivos proporcionados por la producción y la innovación, de manera que se promueve la protección de los activos intelectuales y la ventaja competitiva para las empresas desarrolladoras, por la vía de la restricción de derechos de propiedad, mediante arreglos legales o institucionales (De Laat, 2005). La licencia privativa otorga derechos de explotación económica al desarrollador del software, como forma de individualización y apropiación de la producción, lo cual puede considerarse coherente con el modelo de mercado dominante, en el cual emergen las prácticas del capitalismo cognitivo (Söderberg, 2008). En esta lógica, hacia la década de 1980 muchas empresas empezaron a presionar por la concesión de derechos de propiedad en software (De Laar, 2005). En las luchas por establecer un modelo de software ocurre la concreción de monopolios en el mercado tecnológico, resultado de los intentos por obtener el control de las esferas de producción, uso e intercambio de software. El dominio de buena parte del mercado por parte de grandes corporaciones, como Microsoft, llevó a demandas legales recurrentes por monopolio (Cortés, 2004), como un capítulo de la controversia suscitada.

Mientras tanto, en el caso del software libre, el código fuente está disponible, sin restricciones, para el usuario. En este modelo se piensa la existencia de una comunidad de programadores, los cuales intercambian libremente las piezas de software que producen (De Laar, 2005) sin que la motivación principal para el desarrollo de software sea el lucro (Hess, 2005). Partiendo de la afirmación de que el uso de patentes o derechos de autor es inapropiado para 
promover el desarrollo del software (Stallman, 2004), se presenta la alternativa del software libre como una forma de oposición a lo que se considera un modelo de apropiación de los desarrollos intelectuales que tiene, como consecuencia, la coacción de libertades individuales y sociales (Himanen, 2001; Raymond, 1999). Las licencias de código abierto -antagónicas a las licencias privativas- permiten usar, reparar y modificar libremente el código fuente, además de permitir que las modificaciones se redistribuyan públicamente para su posterior uso, reparación y modificación, en un ciclo continuo de modificaciones y distribución pública del código fuente (De Laar, 2005).

El modelo de producción del software libre ha sido considerado como una forma de entender la tecnología que se aparta de las motivaciones económicas (Stallman, 2004), por tanto, un modo de reivindicación que condujo a la configuración de un movimiento social (Hess, 2005), aunque Caramez \& Ortellado (2011) afirman que las prácticas de desarrollo de software colaborativo anteceden al movimiento software libre, ubicándose como parte de las lógicas de una cultura hacker (Himanen, 2001; Kelty, 2004). La figura de Richard Stallman constituye un referente para la comprensión de la postura política del software libre. Siendo su principal activista, logró la comprensión de esta tecnología como un movimiento social y global, articulado con la emergencia del proyecto GNU, el cual se centra en la propuesta de Licencia Pública General, GPL, base del licenciamiento del software libre (Caramez \& Ortellado, 2011), la cual se institucionaliza con la creación de la Free Software Foundation, FSF, organización influyente dedicada al desarrollo y promoción de software libre.

Stallman (2014) afirma, respecto al software libre, que "no se trata de los precios, que son algo pequeño comparado con lo importante de la libertad de la comunidad. Es un asunto ético del uso de la tecnología". Se hace mención al argumento que afirmaba una diferencia sustancial en los precios de adquisición de las licencias privativas de software, respecto a las de software libre que, en muchas ocasiones, incluso eran gratuitas. Stallman amplía la discusión de los aspectos meramente técnicos y económicos, hacia una reivindicación política de la libertad, la soberanía y la democratización tecnológica en la sociedad del conocimiento. Sobre este debate se movilizó, durante la década pasada, una controversia intelectual muy activa, que elevó las discusiones tecnológicas al campo de la cultura y, desde luego, de la política (Lessig, 2005). El movimiento software libre adquirió una importancia creciente a nivel global, posicionándose como actor crítico del modelo dominante de apropiación privada de las producciones tecnológicas y el control de la información, promoviendo dinámicas de producción opuestas a las formas tradicionales de organización capitalista (Roberts, 2011). Vale mencionar que el panorama dicotómico (software libre/software propietario) cambió en años posteriores con el ascenso de la popularidad de un modelo alternativo, el open source, el cual inicialmente se consideraba parte del concepto más general de software libre, de manera que en la literatura académica aparece, de manera frecuente, el concepto FLOSS, para referir al Free/Libre Open Source Software, incluyendo ambas posibilidades (Meiszner, Glott \& Sowe, 2008; Crowston, Li, Wei, Eseryel \& Howison, 2007).

La alternativa del open source se basa en un modelo de desarrollo de software libre que deja a disposición del usuario el código fuente y su capacidad de modificación, aunque, de manera simultánea, se aleja del discurso reivindicativo del modelo software libre, centrándose en la capacidad mercantil de dicha tecnología. Sobre el asunto señala Stallman (2004):

En 1998, algunas de las personas de la comunidad software libre empezaron a usar el término software de código abierto (del inglés open source software) en vez de software libre para describir lo que hacen. El código abierto se asoció rápidamente con una aproximación diferente, una filosofía diferente, valores diferentes, e incluso un criterio diferente por el cual las licencias son aceptables. El movimiento por el software libre y el movimiento por el código abierto hoy son movimientos separados con visiones y metas diferentes, aunque podamos y trabajemos juntos en algunos proyectos prácticos. La diferencia fundamental entre los dos movimientos está en sus valores, sus formas de mirar el mundo. Para el movimiento por el código abierto, el asunto 
sobre si el software debiera ser de código abierto es una cuestión práctica, no ética (p. 75).

Por su lado, Berry (2006) ha encontrado, en el análisis de los discursos de los movimientos freel libre software y open source, algunos elementos que expresan su identidad, así, muestra que la posición del open source se basa en un discurso relacionado con la eficiencia técnica, asumiendo el software como un problema técnico, en lugar de un problema moral o legal, de manera que la libertad se entiende como la libertad de elección en el sistema de mercado (Berry, 2006). Mientras tanto, el software libre se reafirma como postura política frente al modelo de comercialización no solo del software privativo sino ahora, del open source. En este sentido, el discurso reivindicativo del software libre, en su condición de movimiento social, logra vincularse como propuesta, en las legislaciones tecnológicas en algunos países, particularmente en América Latina.

En el caso europeo, las apuestas por el software libre adoptadas por la comunidad de Extremadura en España y la ciudad de Munich en Alemania, a comienzos de la década anterior, se consideran paradigmas de implementación de políticas en materia de tecnologías libres, las cuales se sostienen en argumentos como la eficiencia tecnológica, la seguridad tecnológica, la independencia tecnológica y la lucha antimonopolio. Se evidencia en tales experiencias el interés creciente de las administraciones públicas por el software libre, expresado en la emergencia de un debate sobre las posibilidades de intervención y promoción de dicha alternativa por parte de los Estados (Reina, 2006). En el caso de Extremadura, el Proyecto Global de Sociedad de la Información, iniciado en 1999, reglamentó el uso del sistema operativo Linux -basado en software libre-, como estrategia de promoción de la sociedad del conocimiento, de manera que se asegurara la conectividad, primero en el sector educativo y, posteriormente, en la Administración Pública (D’Elia, León, Novo \& Otero, 2005).

Así mismo, la experiencia de Munich se considera relevante en la configuración de las controversias sobre el software libre como alternativa tecnológica para el sector público. Recuerda Reina (2006) que, a finales de 2002, el anuncio de Microsoft de suspender el soporte para el sistema operativo NT 4.0, utilizado por la administración local de Munich, motivó alianzas entre sectores políticos para la aprobación de la migración desde el sistema operativo Windows a Linux, lo cual se concretó en los años siguientes, pese a que Microsoft ofreciera al gobierno de la ciudad rebajas en el coste de las licencias de Windows XP y actualizaciones. En otros casos, como el del distrito de Newham, en Londres, se discutió la posibilidad de migración a Linux, pero esta fue desestimada por un acuerdo establecido entre el gobierno local y Microsoft, de manera que "la alternativa del código abierto sirvió como eficaz baza de negociación para modificar las condiciones iniciales de Microsoft" (Reina, 2006, p. 6).

Las experiencias exitosas mencionadas inspiraron propuestas de legislar a favor del software libre en otras regiones. Particularmente, en América Latina, se adoptó la controversia en Brasil y Venezuela, representando casos de éxito en la implementación de software libre para el sector público, mientras que en Perú y Colombia fueron fallidos los intentos de adopción de una legislación en favor de las tecnologías libres. En el caso venezolano, el encuentro entre la revolución chavista y el discurso reivindicativo del software libre favoreció la aprobación de la política de migración al sistema operativo Linux, Decreto 3390 de 2004. Este proceso, sin embargo, no ocurrió de manera inmediata ya que fue hasta el año 2013 cuando la Asamblea Nacional aprobó la Ley de Infogobierno mediante la cual se estableció el uso de software libre por parte de las organizaciones del sector público (Contreras, 2013). Se hace referencia a una cierta resistencia por parte de funcionarios del Estado, para hacer uso de las herramientas de software libre, mientras se promueve su uso bajo los argumentos de seguridad de la información, beneficio económico y desarrollo educativo en general (Roca \& Montilla, 2013).

En Brasil, la difusión del software libre fue promovida por el gobierno del Estado de Rio Grande do Sul en el I Foro Internacional Software Libre $2000 \mathrm{y}$, durante el año siguiente, en el gobierno del presidente Lula, el software libre adquirió un lugar relevante en su movimiento político (Guesser, 2006). Dada la cercanía del gobierno brasilero con 
las ideas del software libre, fue posible la adopción del software libre en la administración pública en los años siguientes, desde la comprensión de esta tecnología como alternativa ante el monopolio y la homogeneización propuesta por la globalización (Dolcemáscolo, 2013). Se establece entonces una política de impulso a la migración hacia el software libre, como estrategia para garantizar la seguridad, la independencia tecnológica y el desarrollo del conocimiento local, entre otros aspectos. Como resultado, se abrió el debate, en varios estados del país, sobre las posibilidades de implementar software libre en todos los órganos de la administración estatal y las escuelas públicas, incluyendo a sectores de la sociedad civil en las discusiones (Guesser, 2006). El uso de software libre en Brasil amplió su alcance tanto en organizaciones públicas como en las empresas, con la oficialización de esta tecnología como opción preferida del gobierno, en consideración de los beneficios que supone la adopción de estándares abiertos (Santos, 2013). La promoción del software libre en Brasil continuó hasta el año 2016 cuando el gobierno propuso reversar su uso y adoptar software privativo de Microsoft, a pesar de que el movimiento software libre ha demostrado gran efectividad, dada su capacidad de influencia y articulación con partidos y políticos a nivel local y nacional, incluso reconociendo sus heterogeneidades y divisiones (Evangelista, 2014). En los casos mencionados el éxito de la política en materia de software libre puede entenderse, en parte, por la articulación entre el movimiento software libre y las políticas de Estado vigentes, en las que se incluyen las prácticas legislativas.

Mientras tanto, en Perú la propuesta legislativa sobre software libre, presentada en 2001, evidenció el interés de la comunidad software libre por ampliar su participación, por vía de la regulación normativa de dicha tecnología. La controversia finalizó con el fracaso de la ley, dada la influencia de actores privados, como Microsoft, empresa que esbozó argumentos en contra de la propuesta al afirmar que representaba un peligro para la seguridad del Estado y los derechos de propiedad intelectual de las corporaciones (Chan, 2004). En 2002 Microsoft otorgó una donación de 550.000 dólares, representados en dinero, software y servicios de consultoría, la cual se produjo "meses después del proyecto de ley de software libre, como un intento de frenar la adopción de software libre en este país" (Estepa, 2007, p. 186). Además, se anunció la traducción del sistema operativo Windows y el paquete Office al idioma Quechua, como parte del acuerdo firmado entre Microsoft y el Ministerio de Educación de Perú. Lo anterior expresa los intereses de esta corporación en la regulación sobre el software en la región, los cuales traducidos en acuerdos con entes políticos favorecieron la legislación a favor del software privativo.

En Ecuador también puede evidenciarse el papel de Microsoft como socio estratégico en la implementación de programas públicos sobre masificación tecnológica, como es el caso de QuitoEduca.Net, el cual se planteó la dotación de equipos de cómputo en las escuelas de la capital (Belén, Bustamante \& Jiménez, 2012). El convenio incluyó la compra de licencias de software a Microsoft y la capacitación de los maestros, como estrategias para la consolidación de la solución de software privativo para este proyecto. En Colombia la implementación del programa Computadores para Educar, propuesto por el estado colombiano como estrategia para la masificación tecnológica, incluyó convenios con Microsoft para su ejecución, inicialmente en forma de donaciones $y$, posteriormente, en compromisos de compra de licencias de sistema operativo y paquetes de software adicionales, de manera que este proyecto fue entendido como un caso de éxito para Microsoft en relación con sus objetivos corporativos (Ministerio de las TIC, 2015).

El debate sigue vigente, si bien, en otros contextos locales el asunto de la regulación del software se considera un problema político relevante. Bouras, Filopolus, Kokkinos, Michalopoulos, Papadopoulos \& Tseliou (2014) han mostrado que la selección de software en el sector público es un proceso altamente político y estratégico, de tal modo, plantean orientaciones políticas para las administraciones públicas europeas, dirigidas al aprovechamiento de los beneficios del software libre, particularmente, el open source. Se afirma que el Free and Open Source software (FOSS) puede ofrecer ventajas al sector educativo, dotando entornos de aprendizaje de bajo costo a través de plataformas y aplicaciones educativas desarrolladas sin la dependencia a proveedores de software propietarios, entre otros 
aspectos. Del mismo modo, se plantea el FOSS como una solución sostenible para los gobiernos, en términos de acceso a licencias y políticas de apertura y accesibilidad de los datos y la información del sector público, entre otros beneficios. Mientras tanto Kim, Shin \& Lee (2015) reafirman el papel central del software en la industria de las TIC, en el análisis de las políticas sobre software en Corea. Hacen referencia a la construcción de un ecosistema tecnológico que soporta el ascenso de la producción de software en el país asiático, como un eje transversal de la nueva economía, lo que implica un asunto de interés público. En el caso coreano se afirma una amenaza para el futuro de las TIC debido a problemas como la baja compensación que obtienen los programadores por su tarea. Esto sugiere un reto para la legislación tecnológica, como base del desarrollo sostenible de software en el competitivo contexto de la economía informacional. La centralidad de la regulación del software constituye, por tanto, un campo de reflexión fundamental para las ciencias sociales.

En el presente documento se plantea un análisis sobre los procesos de controversia suscitados por los proyectos de ley sobre software libre, presentados ante el Congreso de la República en Colombia. Se resalta el papel del movimiento software libre, como promotor de dichas discusiones, en alianza con sectores políticos, desde la comprensión del software como un asunto social, más que técnico. Sobre tal reivindicación es que la comunidad software libre en Colombia logró alinearse en un debate en torno a la posibilidad de regular el software libre como apuesta tecnológica. Las propuestas de regulación del software libre transitaron en torno a los discursos reivindicativos que se presentaron como apuesta para confrontar el modelo dominante capitalista, el monopolio en la producción de software y la reivindicación de las libertades individuales y colectivas frente al ímpetu hegemónico de las grandes corporaciones del sector tecnológico.

\section{Discusión Metodológica}

Para abordar la controversia en torno a una pretendida ley de software libre en Colombia, se planteó una aproximación metodológica en dos momentos: por un lado, un proceso de revisión documental en textos oficiales relativos a los proyectos de ley sobre software libre, presentados en el Congreso de la República y, por otro, una indagación, con una pretensión etnográfica, de la comunidad software libre. La primera fase del estudio se basa en un análisis histórico-documental, mediante el cual se narran algunos episodios sobre las propuestas legislativas presentadas ante el Congreso de la República de Colombia, como proyectos de Ley, los cuales expresan la posición de grupos reunidos en torno a la defensa del software libre y su contraparte. Para esta tarea se consideraron los documentos oficiales publicados en la Gaceta del Congreso, fuente oficial del Estado colombiano, en la cual se recogen los textos de los proyectos de ley presentados y puestos a consideración de trámite legislativo como leyes de la República. En la Gaceta del Congreso, además, se publican documentos relativos al trámite legislativo de los proyectos, así, las posturas del debate expresadas en textos de ponencias a favor y en contra de las posiciones, y la exposición de motivos, como texto justificante de cada proyecto, entre otros.

La revisión de documentos se realizó de acuerdo con la siguiente clasificación: proyectos de ley, exposición de motivos y ponencias negativas. Se planteó un seguimiento a las diferentes posturas que configuraron el debate, en relación con las evidencias textuales mencionadas. Además, se revisaron algunas publicaciones de prensa en las cuales se mencionan aspectos relativos al trámite legislativo de los proyectos, evidenciando la actualidad del tema y su configuración como asunto de interés público. El periodo en el que se ubica la documentación mencionada es la década de 2000-2010, momento en el cual se presentan tres proyectos de ley sobre software libre. Las categorías consideradas para el análisis remiten a los aspectos clave de la controversia, tales como libertades individuales, soberanía tecnológica, seguridad, libre competencia y software libre/software propietario. Aunque, en general, la mirada de los proyectos de ley se abordó en retrospectiva, algunos episodios consideraron el proceso en acción ${ }^{2}$, es decir, en el momento en que cursaba el debate de trámite legislativo. Sin embargo, los textos presentados como base de la controversia expresan solo algunas de sus partes, lo cual constituye una limitación del trabajo. 
Una apuesta adecuada al abordaje de la producción de las regulaciones tecnopolíticas puede encontrarse en el enfoque etnográfico, siguiendo el trabajo de Latour (2010) sobre el Consejo de Estado francés, en el cual se analiza la compleja maquinaria de la institución burocrática moderna, desde lo que llama la elaboración de la ley. Los procesos de construcción de la ley, del derecho, de las normas y de las políticas, bajo el amparo de la legitimidad del Estado, pueden entenderse como modos particulares de enunciación de la verdad. La elaboración de la ley proporciona una descripción material de cómo se alcanzan, registran y ponen en circulación las decisiones (Latour, 2010). Seguir a los actores, en sus prácticas, discursos y tomas de posición, permite la interpretación de las controversias suscitadas en torno a problemáticas específicas. En este sentido, las herramientas etnográficas pueden aportar, de manera significativa, a la comprensión del trabajo diario de la fabricación de leyes, en tanto, la organización y las prácticas, así como los discursos y los aparatos materiales (archivos, etc.), entre otros elementos, constituyen dimensiones simbólicas susceptibles de interpretación.

En el sentido planteado, la segunda fase metodológica intentó una propuesta de corte etnográfico, desde la cual se abordaron las controversias relacionadas con las propuestas de legislar a favor del software libre. Este trabajo se centró en la recopilación de datos principalmente en el espacio de Colibrí, una cuenta de correos que funcionó durante varios ańos como lugar de reunión, socialización y debate sobre asuntos relacionados con el software libre. La participación durante varios años en el espacio virtual de Colibrí permitió la identificación de prácticas de discusión y participación sobre aspectos técnicos, políticos y económicos de interés de la comunidad software libre $\mathrm{y}$, de manera particular, los aspectos relacionados con los proyectos de ley presentados (Colibrí, 2009, Octubre 19). Teniendo en cuenta la propuesta de Beaud \& Weber (2007), se reflexiona sobre las condiciones de la investigación etnográfica, como posibilidad metodológica del trabajo realizado en el trámite de la investigación.

Los autores indican, como primer requisito, que el medio indagado se caracterice por un elevado grado de interconocimiento, esto es, un conjunto de personas que disponen de un cierto número de informaciones nominales y entre las cuales se establecen interacciones. En el caso de Colibrí, la configuración de la comunidad virtual a lo largo de los años se basó en la interacción constante entre múltiples actores (universitarios, programadores, empresarios), entre los cuales existieron vínculos y relaciones de interconocimiento, bien virtuales, bien presenciales, que expresan los acuerdos y desacuerdos sobre asuntos relacionados con concepciones particulares sobre lo tecnológico. En este sentido, la existencia del espacio virtual de la Lista Colibrí es, en sí misma, una expresión del interconocimiento, a pesar de la ausencia de la interacción cara a cara, considerada apropiada en la tradición etnográfica (Hine, 2004). Lo anterior no elimina la posibilidad de construir narrativas o historias sobre la comunidad abordada, dada la interacción y comunicación con los participantes en una cotidianidad online.

Colibrí se hizo posible gracias a que algunos activistas de la comunidad software libre financiaron y apoyaron -durante más de una década- las posibilidades técnicas para la conformación del colectivo, es decir, el uso de servidores y administración requerida para los propósitos de la cuenta digital. La participación de los miembros en la Lista Colibrí se realizó siempre de manera voluntaria, de modo que era posible suscribirse, interactuar y eliminar la suscripción en cualquier momento. En muchos casos la finalización de la suscripción expresaba el desacuerdo o censura respecto a las posturas dominantes en los temas discutidos, lo cual muestra la existencia de disparidades y heterogeneidades al interior de la comunidad software libre. Incluso, parece que el fracaso de los proyectos de ley generó algunas rupturas al interior de la comunidad software libre, las cuales, de algún modo, aportaron a la posterior desarticulación de Colibrí. Mientras la cuenta existió, fue dinámica la interacción en el grupo, así como la emergencia de liderazgos y reconocimientos de aquellos sujetos que dieron voz al colectivo en el marco de los debates legislativos sobre la regulación del software libre en Colombia.

Un segundo aspecto, planteado por Beaud \& Weber (2007), es el de la larga duración, que implica la interacción frecuente, durante periodos de tiempo específicos. La interacción rutinaria entre actores 
involucrados permite los intercambios y la elaboración de enunciaciones y localizaciones. La interacción frecuente con la comunidad software libre en Colibrí -y en otros espacios de encuentro- se generó de manera constante, durante varios años, en los cuales el autor participó. Particularmente, el Festival Latinoamericano de Software Libre, FLISOL, constituye uno de los eventos de difusión del software libre más importante en América Latina, el cual se realiza anualmente en varias ciudades del país, desde 2005 e incluso hasta el presente. El FLISOL ha sido un espacio referente para la comunidad software libre y está dirigido para una diversidad de públicos: académicos, empresarios, técnicos y activistas. Allí se realiza la instalación de diversas distribuciones, o versiones del sistema operativo, basadas en software libre y se organizan talleres y conferencias sobre asuntos relativos al software libre. Durante los ańos en que cursaron los proyectos de ley, los temas que relacionaban política y software libre fueron recurrentes y generaron una dinamización de la comunidad, en la pretensión de construir consensos y representación política que se hiciera visible en los espacios de debate legislativo del Congreso de la República. En el FLISOL se realizaron aproximaciones a actores relevantes de la comunidad software libre, mediante el uso de entrevistas semiestructuradas, orientadas en corroborar las posiciones expresadas mayoritariamente en los espacios virtuales de Colibrí.

Finalmente, se deriva un tercer aspecto, mencionado por Beaud \&Weber (2007), consistente en la reflexividad del proceso, como condición propia del distanciamiento entre el investigador y el objeto, con el fin de identificar estructuras de significación en la comunidad software libre, las cuales no se hacían evidentes, en el contexto de los asuntos discursivos que ocurrieron en Colibrí. Como muestra Hine (2004), el ciberespacio puede ser entendido como un compuesto de textos -más que de interacciones- aunque, tanto la interacción presencial, como virtual, permite pensar en la existencia de estructuras, jerarquías y relaciones de género particulares al interior de la comunidad (Pérez-Bustos, 2010). La necesidad de tomar distancia de las posturas de la comunidad software libre, expresadas en los proyectos de ley, fue un aspecto clave para identificar elementos centrales en la controversia e interpretar las dinámicas que enmarcaron los procesos analizados. Un aspecto clave a este respecto es el de la consideración de la ciberetnografía -o etnografía virtual-como posibilidad para el abordaje de Colibrí. Ya se han mencionado los criterios propuestos por Beaud \& Weber (2007), aunque hace falta decir que el espacio de Colibrí fue entendido, no desde una demarcación estrictamente territorial sino como un lugar simbólico y sociocultural, susceptible de la aproximación etnográfica, si bien es allí en donde se producen las relaciones e interacciones objeto de observación. Así, se trató de estudiar a la comunidad en el ciberespacio (Ruiz, 2008, p. 129).

El ciberespacio, sin duda, permite el análisis de grupos en los cuales es difícil encontrar participantes en la realidad presencial, como es el caso de Colibrí, lugar que favoreció el encuentro de actores diversos en una interacción usualmente asincrónica. En la comunidad virtual Colibrí están presentes las personas, tienen continuidad, se pueden identificar sus tránsitos y sus posiciones, en la medida en que un asunto de interés público logra convocarlos y generar un debate continuo que se extiende en el tiempo y se configura adquiriendo una cierta personalidad. Las posiciones consensuadas permiten dar voz a los actores ya que se establecen como miradas de la comunidad, expresadas posteriormente en los ámbitos legislativos en los cuales se discutieron los proyectos de ley, en una contraposición de la postura del Estado, la cual mostró un interés por desvirtuar los argumentos presentados por el movimiento software libre, en cabeza de sus líderes. Vale mencionar que la posición del Estado, como actor de la controversia, fue validada desde los textos presentados y publicados en la Gaceta del Congreso, ya que no hubo acercamiento a los legisladores o políticos que promovieron las ponencias en contra de los proyectos presentados, aspecto que representó una limitación adicional para el análisis. La validez de Colibrí, como espacio para la observación y recolección de datos puede justificarse, además, reafirmando la condición de la cultura hacker, en su actuación online (Aibar, 2008), de tal modo que entender un colectivo cuyas interacciones sociales están mediadas fundamentalmente por la virtualidad, implica tratar de entender sus prácticas en los tránsitos del ciberespacio, así, los modos de vida de la red (Hine, 2004). 
A pesar de que la lista Colibrí deja de funcionar hacia el año 2014, el registro de la actividad puede seguirse en archivo online. Tanto en sus tránsitos como en la aproximación documental de los registros del archivo de Colibrí, se consideró el espacio virtual como un lugar simbólico y sociocultural, en tanto su capacidad de movilizar procesos de comunicación, generados en interacciones constantes, otorgando sentido a la participación de los diversos miembros. El espacio de Colibrí expresa, por lo menos en el discurso, las posiciones de los actores, sus intereses y quimeras, como una postura política frente a la realidad. Si bien, en el contexto de la comunidad software libre se encuentran matices que involucran tanto posiciones propias del movimiento software libre, como aquellas que se sustentan en la lógica del open source, se reafirma la legitimidad de prácticas cooperativas de producción de conocimiento, asociadas con las lógicas de producción de software libre, aspecto que otorgó identidad y cohesión al colectivo (Barrio, 2008).

\section{Proyectos de ley sobre software libre en Colombia}

Durante la década de los 90 se alinearon en Colombia diversos actores en torno al software libre. En el contexto universitario estudiantes y programadores plantearon iniciativas, a nivel nacional, organizándose en una veintena de grupos defensores o promotores de dicha tecnología. El software libre logró constituirse en tendencia en contextos académicos (Delgado \& Cuello, 2007), principalmente en el ámbito de facultades de ingeniería, en donde se articuló con discursos que reivindicaban la lógica del hacker, propia de personas dedicadas de manera entusiasta a la programación y cuyas motivaciones distaban del interés económico (Himanen, 2001). En torno a la tarea técnica se movilizó un asunto político, lo cual facilitó la consolidación de la comunidad software libre, de manera similar a lo ocurrido en otros países, constituyéndose el software libre en un tipo de technology -and product-oriented movements (TPMs), esto es, movilizaciones de la sociedad civil -muchas veces en alianza con sectores privados- que promueven el cambio social mediante el apoyo a tecnologías alternativas y a políticas asociadas con las mismas (Hess, 2005).

En el caso colombiano la comunidad software libre diseñó un espacio de encuentro, la lista Colibrí, el cual desde el 2002 agrupó un número de técnicos, usuarios, académicos y activistas del software libre, entre otros, quienes tuvieron la posibilidad de interactuar en las líneas de discusión, generadas en relación con los temas de interés para dicho colectivo. En el contexto virtual de Colibrí se expresaron diversos asuntos relacionados con una propuesta para la regulación del software libre, de manera que permitiera hacerlo visible como alternativa para el Estado, frente al modelo dominante de software privativo. Las alianzas entre la comunidad software libre y sectores políticos dinamizaron las propuestas, las cuales, posteriormente, se concretaron en proyectos de ley presentados ante el Congreso de la República. Durante una década tres proyectos de ley sobre software libre fueron planteados, el primero de ellos, Proyecto de Ley 83 de 2002 "por medio del cual se incentiva el uso del software libre como mecanismo para fomentar el respeto a los derechos constitucionales de los ciudadanos e incentivar el desarrollo tecnológico de la nación" se presentó ante la Cámara de Representantes, por parte del congresista de izquierda Gustavo Petro, como resultado del trabajo colaborativo con líderes y activistas de la comunidad software libre. En el contexto del Seminario GNU/Linux, liderado por el grupo de software libre EIDOS de la Universidad Nacional de Colombia, se hace pública la presentación del proyecto, reivindicando el papel de la comunidad universitaria en su construcción.

La justificación principal del proyecto fue la posibilidad del software libre como mecanismo para garantizar los derechos constitucionales, desde la lógica de democratización de la información, siguiendo experiencias -exitosas- como las de Munich y Extremadura. En la exposición de motivos se afirmaba que "el movimiento del software libre es una tendencia global que, de ser aprovechada de manera apropiada, puede impulsar significativamente el progreso tecnológico del país al darle verdadero control de los sistemas de información que utiliza y de los que depende" (Congreso de la República, 2002). Se adscribe al discurso que se popularizó en el 
momento, en el cual se lanzaba una crítica al modelo de software propietario por la imposibilidad de sus usuarios de mantener el control sobre la información bajo dicho esquema de licenciamiento, además de la incapacidad del Estado para garantizar el acceso de los ciudadanos a los documentos y la información correspondiente al sector público, por tanto, de interés colectivo (Gómez, 2003). De tal modo, se afirmaba la importancia de tener el control del software utilizado en la infraestructura del Estado como mecanismo para garantizar la seguridad nacional y la calidad de vida de los ciudadanos (Congreso de la República, 2003).

La reivindicación de la libertad aparece en el Proyecto como justificación para la legitimidad del software libre frente a la limitación de dichas prerrogativas por parte del modelo propietario. Como indica González (2003), la legislación sobre propiedad intelectual, por legítima que sea, constituye "probablemente la mayor intromisión en la libertad individual que tenemos en las sociedades democráticas" (p. 2), considerando el papel relevante de la información, como bien fundamental de la sociedad del conocimiento. Desde tal supuesto, el proyecto de ley de 2002 hizo suyo el argumento de la defensa de la libertad, mostrando al software libre como una tecnología inherentemente política, es decir, un sistema ideado por humanos, que parece ser fuertemente compatible con ciertos tipos de relaciones sociales o, incluso, encarnando relaciones de poder (Winner, 1992). De tal modo, se pretendió dar coherencia al software libre con un discurso legitimador de los intereses particulares del movimiento software libre, los cuales podían extenderse a la ciudadanía, en general. Se incluye en el proyecto de ley el texto de las cuatro libertades que definen el concepto de software libre: (1) libertad de usar el programa, con cualquier propósito, (2) libertad de estudiar cómo funciona el programa y modificarlo, adaptándolo a las propias necesidades, (3) libertad de distribuir copias del programa, con lo cual se puede ayudar a otros usuarios y (4) libertad de mejorar el programa y hacer públicas esas mejoras a los demás, de modo que toda la comunidad se beneficie ${ }^{3}$.

Remitir a los principios establecidos por Stallman fue un común denominador en diversos proyectos de ley y aprobaciones legislativas en las experiencias internacionales mencionadas. En base a las cuatro libertades, el Proyecto argumentó que la propuesta libre para el desarrollo del software en Colombia apuntaba a la reivindicación de derechos constitucionales en la consideración, como principios de la ley, de las responsabilidades del Estado respecto al control efectivo sobre los sistemas de información -de los que depende su funcionamiento-además de la promoción de la igualdad al acceso de la información por parte de los ciudadanos, evitando depender de proveedores únicos (Congreso de la República, 2002). El argumento se sostiene en la afirmación de que el software libre necesariamente garantizaría las libertades ciudadanas y la seguridad del Estado en tanto que el código fuente al estar a disposición del público, favorecería la transparencia y el acceso a la información. Además, se afirmaba que el Estado carecía de la capacidad de garantizar el acceso a la información por parte de los ciudadanos en el caso de los documentos públicos almacenados en formatos basados en software propietario, ya que "al no tener acceso al código fuente, los usuarios del software, en este caso las entidades del Estado no pueden estudiar su operación, únicamente el proveedor de software libre conoce la manera en que se lleva a cabo su labor. En los casos en que los proveedores de software propietario insertan en él funcionalidades escondidas de propósitos dudosos, suele ser difícil que los usuarios lleguen a ser conscientes de su existencia" (Congreso de la República, 2002, p. 3).

La contraposición de la postura planteada por la comunidad software libre, se expone en la ponencia negativa presentada por la Representante a la Cámara Rocío Arias, en la cual se anuncia que el proyecto viola principios constitucionales "al poner en riesgo el ejercicio mismo de la función pública obligando a las entidades a adquirir software que puede resultar ineficiente, inseguro o incompatible con las funciones" (Arias, 2003). De tal manera, se pone en duda la capacidad técnica del software libre en la garantía de la seguridad y eficiencia del Estado. Por otro lado, a pesar de promover la democratización en el acceso de la información el Proyecto propone en su Artículo 4 o lo siguiente: "todas las instituciones del Estado y las empresas donde el Estado posea mayoría accionaria emplearán exclusivamente software libre en sus sistemas de información” (Congreso de la República, 2002, 
p. 3). Lo anterior fue entendido por los opositores del proyecto como una contradicción respecto al no monopolio de los proveedores de software, generando el principal argumento en contra para el archivo de la ley en su primer trámite legislativo.

Señala Arias (2003) al respecto: "lo que vemos, a primera vista, en este proyecto es la imposición al Estado de una camisa de fuerza que lo obliga a adquirir y utilizar sólo determinado tipo de software, quitándole la opción legítima de elegir otro que pueda ser más eficiente $\mathrm{o}$ adecuado para su funcionamiento en un momento dado". Sobre el análisis de constitucionalidad se afirma una vulneración a la libre competencia dado el propósito de crear un monopolio, del mismo modo, en términos de los principios de contratación estatal, se afirma que el proyecto de ley viola el principio de igualdad entre los posibles licitantes, por tanto, de la transparencia en este tipo de procesos (Arias, 2003). Finalmente se llega a afirmar, en la ponencia negativa, que el software libre conlleva más riesgos que ventajas e incluso, que no representa una disminución del gasto dados los costos de mantenimiento, adaptación y capacitación a los usuarios, por tanto, negando los argumentos más recurrentes defendidos por la comunidad software libre.

$\mathrm{El}$ argumento técnico se hizo recurrente en el contexto de Colibrí, reafirmándose los alcances políticos del software libre. Lo anterior se expresa en la recurrencia de la postura sobre el monopolio vigente en el modelo de software privativo, cuya evidencia principal era la tradición de contratación pública del Estado con corporaciones globales como Microsoft (Colibrí, 2014, Junio 24), en el contexto de las políticas y acciones relacionadas con la popularización de las tecnologías informacionales en sectores públicos relevantes, como la educación. Una vez archivado el Proyecto de Ley de 2002, se mantuvo el debate en el contexto de colibrí durante los años siguientes, sobre el fracaso del proyecto. Uno de los principales activistas de la comunidad Colibrí (2011, Marzo 24) afirma:

La ley de software libre no fue presentada a debate legislativo en el 2002 a pesar de estar completa, en el sentido de su redacción. Eso se debió a una insistente campaña de lobby por parte del gremio de software, que supuestamente representaba a todos, pero que claramente está al servicio de los monopolios privados. Estas acciones de lobby se vieron reflejadas en coincidenciales encuentros en los pasillos del Congreso con algunos de los directivos de dicho gremio, que no dejan más vestigios que lo anecdótico, pero que quienes lo vivimos en primera persona, podemos dar cuenta de la coincidencia y cómo esta afectó el desarrollo de la presentación de la ley.

Hace referencia el comentario a las alianzas que, al parecer, se establecieron entre empresarios del software y políticos, con el fin de favorecer una determinada regulación en la materia. Como sucedió en otras experiencias, de acuerdo con lo ya mencionado, en el caso de los proyectos de ley sobre software libre en Colombia se hizo evidente la articulación de intereses entre legisladores y empresarios, práctica que ha definido en muchos casos el curso de la reglamentación en materia tecnológica a favor de intereses específicos. El papel del lobby empresarial, aunque no es un tema central en el presente trabajo, representa una dimensión explicativa sobre las dinámicas de construcción de la política tecnológica en relación con el software libre y se constituye en uno de los argumentos centrales que explica el archivo del Proyecto, desde la perspectiva de la comunidad software libre, la cual continúa durante los años siguientes discutiendo la posibilidad de la Ley.

En el ańo 2007 fue presentado ante el Congreso de la República un nuevo intento para la legislación del software libre. Se trata del Proyecto de Ley 021 de 2007 "por el cual se implementa la utilización del software libre en las entidades del Estado", radicado el 26 de Julio en la Comisión Sexta de la Cámara de Representantes por tres parlamentarios del partido MIRA, un movimiento político independiente adscrito a la Iglesia Pentecostal, evidenciándose un cambio en las alianzas realizadas entre la comunidad software libre y los partidos políticos que, a la vez, modifica el enfoque del nuevo proyecto. En el caso del proyecto de Ley 83 de 2002 existía una reclamación anticapitalista respecto a las prácticas de producción y comercialización del software, coherente con la ideología del partido político que lo 
lideró, lo cual cambia en el proyecto de Ley 021 de 2007. La reconstitución de los actores, en términos de nuevas alianzas entre la comunidad software libre y sectores políticos, deja por fuera discursos que reflejan la resistencia del software libre frente al modelo propietario dominante. De hecho, uno de los aspectos más significativos en el curso del Proyecto de Ley de 2007 fue precisamente las modificaciones que se realizaron antes del Primer Debate, a partir de las cuales se cambió incluso el título mismo de la Ley. Se puede hablar entonces de dos textos, el que se presenta inicialmente el 20 de Julio de 2007, muy cercano en su texto e intencionalidad al Proyecto de 2002, y un segundo texto modificado que no pretende regular específicamente el software libre.

El Proyecto de Ley 021 de 2007 respecto a su antecedente de 2002 tuvo un mayor eco ya que, si bien fue archivado, alcanzó a llegar a un segundo debate en el tránsito legislativo. Como afirma A. Castelblanco, uno de los miembros más activos de la comunidad Colibrí y promotor de la ley de software libre en aquel momento: "este es el proyecto que mayor alcance ha tenido en el gobierno" (Colibrí, 2011, Marzo 24), incluso actualmente la ley de TIC en Colombia recogió algunos párrafos del proyecto construido sobre el software libre en este momento (Comunicación Personal, Abril 11 de 2016). Buena parte del texto del proyecto fue redactado por Castelblanco junto con Jazmín Olarte, Representante a la Cámara por el Partido MIRA y ambos enfrentaron el debate en la Cámara de Representantes "invitando a algunos compañeros de la comunidad a participar (primero con sus observaciones y posteriormente con su voz en el debate público)" (Colibrí, 2011, Marzo 24). Esto evidenciaba un trabajo colectivo, entre la comunidad software libre y el partido MIRA, alianza que contaba con un importante y creciente número de seguidores. Olarte, interactuando en la lista Colibrí, invitaba a los participantes a la audiencia pública sobre el proyecto de ley de software libre que se realizó en octubre (Colibrí, 2007, Septiembre 26) y aclaraba apartes del proyecto en una constante interacción con la comunidad, la cual participó, por medio virtual, de manera dinámica en los debates suscitados.

El Proyecto de 2007 se presenta inicialmente como un segundo intento de la iniciativa de 2002, teniendo como punto de partida la ideología de las cuatro libertades del software libre. El texto aborda el asunto de la independencia tecnológica y otros aspectos relacionados con el acceso y la regulación del uso tecnológico, así, en el Artículo 2 se indica: "El objeto de esta ley es incentivar el desarrollo tecnológico de las entidades públicas en todo el territorio nacional, para que tengan un verdadero control de los métodos que utilicen, y puedan organizar sus sistemas informáticos de manera eficiente y productiva. El Estado garantizará que sus entidades no dependan de servidores únicos de Software, promoviendo la igualdad de acceso a este tipo de tecnología" (Congreso de la República, 2007). Básicamente el Proyecto planteaba la expedición de una política de promoción y uso de software libre, estableciendo 11 aspectos mínimos que favorecieran la lógica de inclusión del software libre, entre los cuales aparece el apoyo a proyectos de investigación y desarrollo de software libre para entidades del Estado, la capacitación y el fomento de la cultura de uso de software libre y propiciar el uso compatible y la convivencia de software libre con sistemas propietarios que se encuentren en ejecución en diferentes sectores. El primero de los 11 aspectos citados apunta a "la definición de procedimientos y el empleo de estándares abiertos que permitan la interoperabilidad entre los sistemas propietarios heredados y el software libre" (Congreso de la República, 2007). Se incluye, entre tanto, una perspectiva moderada sobre los procesos de implementación de software libre, contraria al intento impositivo del primer proyecto.

En el Artículo 60 se indica: "las áreas de sistemas de todas las instituciones públicas y privadas de educación formal en el país promoverán la enseñanza del software libre, a la par con la enseñanza del software propietario" (Congreso de la República, 2007), lo cual plantea una tarea de promoción del software libre -por la vía educativa- pero difícil de realizar considerando la estructura de las redes informáticas del sector educativo atadas a soluciones privadas ${ }^{4}$. En la exposición de motivos del texto se citaban ventajas técnicas: "los expertos consideran a Linux como un sistema operativo muy estable [...] el software libre es muy robusto y en comparación con el propietario suele tener menos errores de programación que lo 
vuelvan vulnerable a ataques de seguridad, virus, worms y otros problemas que afectan constantemente a la mayoría de programas propietarios", y argumentos políticos: "el software libre también es una herramienta que busca el equilibrio social, al darle oportunidad al usuario de adaptar el sistema a su necesidad" (Congreso de la República, 2007). Como justificación, se mencionan las experiencias internacionales de software libre consideras "exitosas" y el impacto fiscal en términos de ahorro del Estado por concepto de licencias de software

No obstante, el texto original es modificado para presentarse en Primer Debate ante la plenaria de la Cámara de Representantes. Ya no enfatiza en los aspectos relativos al software libre como eje central de la ley sino al asunto de la regulación tecnológica en el contexto del marco económico global. Entre los aspectos centrales del documento presentado como Ponencia para el Primer Debate al Proyecto de Ley 021 de 2007 se encuentra el reconocimiento de las tendencias internacionales en materia tecnológica y la consideración de las tecnologías como mecanismos para promover el desarrollo económico. En la exposición de motivos se lee, a propósito de la declaración de la ONU conocida como Objetivos del Milenio: "uno de los objetivos acordados es fomentar una asociación mundial para el desarrollo, la cual incluye dentro de sus estrategias, velar en colaboración con el sector privado porque se puedan aprovechar los beneficios de las nuevas tecnologías, en particular, los de las Tecnologías de la Información y las Comunicaciones" (Congreso de la República, 2008). Así que se adoptan estas estrategias mediante la regulación del software libre en torno a problemas centrales como el mejoramiento de la educación en Colombia y la reducción de la brecha de apropiación de las TIC, desde una mirada determinista que sugiere a las TIC como factor fundamental "para que las generaciones presentes y futuras estén mejor informadas, tengan mayores oportunidades de empleo y puedan acceder a los beneficios de una sociedad interconectada" (Congreso de la República, 2008).

Bajo el argumento de la neutralidad tecnológica, se evita la mención al tratamiento preferencial del software libre, incluso se elimina del título: Proyecto de Ley 021 de 2007 "por la cual se fijan políticas y establecen criterios para la administración y adquisición de programas de computación por parte del Estado". En el Artículo 3, incluido en la ponencia, "se establece que para la adquisición de software en las entidades estatales, el Jefe de Sistemas o quien haga sus veces, deberá establecer las necesidades específicas del software requerido, tomando en cuenta, además de las opciones propietarias, las que se conozcan como software libre, si para el efecto existen" (Congreso de la República, 2008). En el primer debate del Proyecto de Ley, realizado el 5 de diciembre de 2007, se realizaron algunas modificaciones fundamentales, así, se cambia el texto "tomando en cuenta además de las opciones propietarias, las que se conozcan como software libre" por "teniendo en cuenta la oferta de tecnologías de la información y telecomunicaciones existentes en el mercado debidamente certificadas", esto con el fin de reafirmar los principios de equidad que pusieran en el mismo nivel a las diversas soluciones existentes en el mercado. El texto de Ponencia para Segundo Debate afirma en sus objetivos que, el nuevo articulado "no busca hacer obligatoria la adopción del software libre sino, solamente, incentivar su desarrollo y utilización en entidades públicas por considerar que puede representar un ahorro para el fisco" (Congreso de la República, 2007). Evidentemente estas modificaciones se consideraban necesarias en consideración de los vicios de inconstitucionalidad de los que se acusó al Proyecto de Ley de 2002.

En el texto de Ponencia para Segundo Debate presentado ante la Comisión Sexta Constitucional de la Cámara de Representantes se presenta una nueva modificación al Proyecto de Ley 021 de 2007 "por el cual se fijan políticas y se establecen criterios para la administración y adquisición de programas de computación por parte del Estado" (Congreso de la República, 2008). El texto presentado planteó, como parte de sus principios, los asuntos relativos a la regulación tecnológica en la cual el tema del software libre no necesariamente implicaba un aspecto relevante o novedoso. C. Botero, miembro de la fundación Karisma y activista del software libre, afirmaba en momentos previos a la votación del Proyecto en el segundo debate: 
Del proyecto original no quedó sino el nombre frente al texto que está ahora disponible para votación en la Plenaria de la Cámara de Representantes. Efectivamente en la comisión el texto se cambió por uno en que se definen unos principios, se aborda el tema de apropiación de Tecnologías de la Información y las Comunicaciones (TIC) y se aprovecha para ocuparse en varios artículos de COINFO (Comisión Intersectorial de Políticas y de Gestión de la Información para la Administración Pública), sin hacer ninguna referencia al software libre, a sus apuestas, ni a sus principios" (Botero, 2007).

Evidentemente el Proyecto de 2007 abandonó su asunto central, el software libre, para centrarse en aspectos de regulación tecnológica no necesariamente relacionados con su tema central inicial. Algunas discusiones relacionadas con este proyecto se mantuvieron posteriormente en otras propuestas, de allí que A. Castelblanco haya afirmado que fragmentos del Proyecto se encuentren presentes en la Ley de TIC sancionada por el Congreso de la República en el año 2009.

En 2009 se presentó, sin embargo, una nueva propuesta por el partido MIRA, planteándose como objeto de la Ley la pretensión de "incentivar el desarrollo tecnológico en todo el país, lo cual va a permitir garantizar un control efectivo de métodos que las mismas entidades utilicen, permitiendo que se pueda organizar sus sistemas informáticos de manera eficiente y productiva" (Mira, 2009). En el documento de ponencia para primer debate, presentada en agosto de 2009 ante la Comisión Sexta de la Cámara de Representantes, se afirma que el texto corresponde, en parte, al del proyecto 021 de 2007 agregando que este último "dio inicialmente una idea errada del mismo, puesto que pudo ser interpretado como la intención de establecer por ley la obligatoriedad de la implementación del software libre en las entidades estatales. Esa, sin embargo, no fue la intención de los autores" (Congreso de la República, 2009). De allí que en la nueva propuesta se aclara que la pretensión no corresponde con hacer obligatoria la adopción del software libre, argumento que deslegitimó el Proyecto 083 de 2002, sino que el espíritu que anima la propuesta es el de "solamente, incentivar su desarrollo y utilización en entidades públicas por considerar que puede representar un ahorro para el fisco" (Congreso de la República, 2009).

Logra consolidarse, entonces, una postura moderada, alejada de la propuesta de sectores radicales de la comunidad de software libre que optaban por una reglamentación tendiente a una migración obligante de los sistemas propietarios del Estado a sistemas libres, específicamente, sistemas operativos Linux. El texto de ponencia del Proyecto de Ley 017 de 2009 reconoce que el cambio en el título del proyecto, que ya no mencionaba de manera específica al software libre, era una condición fundamental para legitimar la perspectiva real de la propuesta. Algunos foristas, en el debate realizado en la cuenta Colibrí, entendieron el asunto como un alejamiento del proyecto de los intereses de la comunidad software libre, no obstante que el Proyecto de Ley 017 de 2009 retomara en su Artículo $1^{\circ}$ las definiciones relacionadas que delimitaban en las dos primeras iniciativas legislativas los conceptos de Software, Código Fuente, software libre, Software Privativo o Propietario e, incluso, la referencia a las cuatro libertades que definen el software libre como filosofía.

En el documento presentado para primer debate se plantea como justificación la necesidad de que el Estado regule la tecnología, considerando su presencia cada vez mayor en los contextos sociales, dado el avance imparable de la tecnología (Congreso de la República, 2010). En tales términos se remite a asuntos relacionados con la regulación tecnológica, sin una relación directa con el uso de software libre respecto a otras soluciones, al igual que ocurrió en el Proyecto de 2007. La idea de incentivación al desarrollo y la promoción a la adopción de herramientas tecnológicas gira en torno al concepto de Neutralidad Tecnológica, relacionándola, a su vez, con la obligación del Estado de garantizar derechos como la igualdad de las personas ante la ley, el derecho a la educación y el acceso a la información, la libre competencia y la gestión de la información por parte de los ciudadanos al amparo de la seguridad y la soberanía tecnológica. Además, se hace referencia a la posibilidad de construir una dimensión digital del Estado, considerada relevante en el marco del fortalecimiento de la industria de 
las tecnologías informacionales, la ciberseguridad (Martínez, 2018) y otros asuntos con presencia amplia en las relaciones sociales actuales. Finalmente, no existen variaciones importantes en este nuevo intento de Proyecto de Ley sobre software libre que, como ya se mencionó, es una propuesta por la regulación del software y las tecnologías de la información y la comunicación más que una forma de legitimar o deslegitimar el software libre propiamente.

En el texto de Ponencia para el Segundo Debate del Proyecto de Ley 021 de 2009, presentado en junio de 2010, se aclara la importancia del software en el contexto de la Sociedad de la Información y la definición de software libre como mecanismo para lograr independencia y control sobre la información. Se modifica el Art. $2^{\circ}$ del anterior texto con el propósito de aclarar que "la adopción y uso de la tecnología del software responda esencialmente a las necesidades del país en general y de las instituciones oficiales en particular, antes que a otros criterios" (Congreso de la República, 2010). Finalmente se reafirma como intención la "Priorización y promoción del uso de software, atendiendo fundamentalmente a las necesidades del desarrollo nacional e institucional, a criterios de calidad, variedad y ventajas competitivas de mercado en el país" (Congreso de la República, 2010), sin dar preferencia a ningún tipo de software, simplemente atendiendo a una política de uso de software que responda a criterios de calidad, variedad y ventajas competitivas del mercado. El proyecto de Ley 021 de 2009 fue archivado en segundo debate, debido, entre otros argumentos, al concepto de la Corte Suprema de Justicia que sostuvo que el concepto de neutralidad tecnológica va en pro del libre mercado y la competencia (Colibrí, 2007, Noviembre 13). A pesar de esto, el Proyecto de Ley 021 de 2009 logró reafirmar la necesidad de promover una política de uso del software en Colombia.

\section{Consideraciones Finales}

Los intentos de legislar el software libre en Colombia y, de manera más amplia, en América Latina, constituyen episodios sobre la construcción de la política tecnológica y sus implicaciones para el orden social. Hacen evidente la relevancia de las relaciones tecnopolíticas y sus regulaciones para el desarrollo regional, lo cual se expresa en la participación ciudadana en las controversias suscitadas, la emergencia de movimientos sociales y políticos en dicho contexto y las comprensiones contemporáneas sobre las relaciones entre Estado y ciudadanía. El tránsito de los proyectos de ley sobre software libre en Colombia expresan las luchas por la inclusión de esta tecnología en los escenarios de la regulación estatal, las cuales logran movilizar posiciones divergentes frente al modelo dominante de producción, uso y comercialización de software pero, sobretodo, evidenciar las prácticas de una política tecnológica alineada con la lógica de control por parte de grandes monopolios del sector tecnológico, los cuales han logrado, de cierto modo, imponer sus condiciones mediante la articulación con las políticas nacionales. Las prácticas del lobby empresarial en el contexto de las legislaciones puede ser clave de interpretación de muchas de las dinámicas que se presentan en la construcción de la ley, aunque no sean evidentes desde la aproximación documental a los tránsitos de la producción legislativa, en relación con los documentos oficiales que refieren específicamente a los proyectos de ley y los debates que cursan en los procesos de discusión. Una tarea más amplia en el propósito de interpretar las alianzas que se establecen en los procesos de construcción de la ley implicaría abordar desde una aproximación etnográfica los procesos y actores involucrados, además de una revisión de documentos relacionados con la contratación pública.

Por el momento, se ha evidenciado el privilegio que se otorga al modelo de software privativo en la legislación tecnológica colombiana, pese a la masificación actual del sistema operativo Android (basado en Open Source) instalado en la mayor parte de dispositivos electrónicos de uso personal en el mundo y la presencia de sistemas operativos Linux en el segmento de servidores. El software libre, en todo caso, ha logrado entrar en los escenarios de producción y uso de dispositivos tecnológicos, aunque las discusiones filosóficas o políticas que lo sostuvieron perdieran su relevancia o dinamismo inicial. El fracaso de los proyectos de software libre en Colombia condujo a una cierta desarticulación de lo que podía considerarse un movimiento social, la 
comunidad software libre. Debido a la multiplicidad de intereses internos, pero particularmente, la imposibilidad de alcanzar las prerrogativas de los activistas de dicha tecnología, muchos grupos se dedicaron al desarrollo en ambientes basados en open source y a la creación de empresa, o bien, migraron a otro tipo de desarrollos tecnológicos. Sin embargo, las ideas que han sostenido al movimiento software libre no se extinguen, sino que transitan a nuevos ámbitos en los cuales permanecen las discusiones sobre la defensa de la libertad y la soberanía tecnológicas, entre otros asuntos actuales. La desaparición de Colibrí puede expresar la desarticulación del movimiento software libre, la cual se evidenció, además, con la desaparición de la gran mayoría de grupos universitarios e institucionales que promovieron el software libre durante varias décadas, concretamente de sus espacios virtuales en la web. No obstante, las luchas por la reivindicación de la libertad del Internet, la inclusión tecnológica y la disminución de la brecha digital, se mantienen vigentes en el escenario de la tecnopolítica regional y mundial. Nuevos proyectos liderados por antiguos miembros de Colibrí, como es el caso de Bogota Mesh, entre otros, muestran la permanencia de los argumentos en favor de las tecnologías libres, como una tarea vigente y urgente en América Latina.

\section{Notas}

1 El código fuente es un conjunto de instrucciones que permiten la ejecución de un programa de computador, expresadas en código binario, por tanto, entendidas por el ordenador. Para ser puestas a disposición del usuario, se requiere un proceso de compilación -o traducción- a un código susceptible de comprensión por parte de este.

2 Se hace referencia a la obra de Latour, Ciencia en Acción, en la cual se sigue a los actores, en este caso los científicos e ingenieros, en el proceso de comprensión de la ciencia, no en sus resultados, sino en términos de las controversias que emergen en el proceso mismo de construcción de los hechos científicos. Ver Latour (1992) y Latour \& Woolgar (1995).

3 Las cuatro libertades constituyen una serie de prerrogativas que definen el software libre, el concepto fue establecido por Richard Stallman, activista y miembro fundador de la Free Software Foundation, organización creada en Estados Unidos hacia 1985 con la idea de promover el software libre, su filosofía y los aspectos técnicos que lo definen, principalmente, los tipos de licencia que representan al software libre, particularmente la Licencia Pública General, GNU.

4 Para citar un caso, la plataforma tecnológica de la Secretaría de Educación de Bogotá, RedP, se encuentra soportada, desde hace más de dos décadas, en sistemas basados en Windows con compromisos de compra de licencias a varios años.

\section{BIBLIOGRAFIA}

AIBAR, Eduard. (2008). Las culturas de internet: la configuración sociotécnica de la red de redes. Revista CTS, 11, 4: 9-21.

ANDRADE, Jesús \& CAMPO-REDONDO, María. (2012). Apropiación social de las tecnologías de información: políticas públicas para la participación ciudadana. Quórum Académico, 9, 1: 52-68.

ARIAS, Rocío. (2003, Abril 22). Ponencia Negativa presentada por la Representante a la Cámara Rocío Arias del Departamento de Antioquia. Recuperado de http://igor.tamarapatino.org/ proyectoley/Negativa.txt

BARRIO, Alonso. (2008). La apropiación social de la ciencia: nuevas formas. Revista Iberoamericana de Ciencia, Tecnologia y Sociedad, 4, 10:213-225.

BERRY, David. (2006). The contestation of code. A preliminary investigation into the discourse of the free/libre and open source movements. Critical Discourse Studies, 1, 1: 65-89.

BELÉN, María; BUSTAMANTE, Mónica \& JIMÉNEZ, Javier. (2012). Computadores y Cajas Negras. Quito, FLACSO.

BOELLSTORFF, Tom; NARDI, Bonnie; PEARCE, Celia \& TAYLOR, T. (2012). Ethnography and Virtual Words. A Handbook Method. New Jersey, Princenton University Press.

BOTERO, Carolina. (2007, Diciembre 16). El que era el proyecto de software libre en el Congreso Colombiano. Recuperado de https://karisma. org.co/carobotero/index.php/2007/12/16/ el-que-era-el-proyecto-de-software-libre-en-elcongreso-colombiano/ 
BOURAS, Christos; FILOPOLUS, Anestis; KOKKINOS, Vasileios; MICHALOPOULOS, Sotiris; PAPADOPOULOS, Dimitris \& TSELIOU, Georgia. (2014). Policy recommendations for public administrators on free and open source software usage. Telematics and Informatics, 31, 2: $237-252$.

BEAUD, Stéphane \& WEBER, Florence. (2007). Guia para a pesquisa de campo. Produzir e analizar datos etnográficos. Petrópolis, Editora Vozes.

CARAMEZ, Maria \& ORTELLADO, Pablo. (2011). Activist-driven innovation: uma história interpretativa so software livre. Revista Brasileira de Ciências Sociais, 26, 76: 77-102.

CASELLS, Manuel. (2002). La Era de la Información. Economía, Sociedad y Cultura. La Sociedad Red. México, Siglo XXI.

CASTORIADIS, Cornelius. (2013). La institución imaginaria de la sociedad. México, Tusquets Editores.

CEPAL. (2013). Economía digital para el cambio estructural y la igualdad. Santiago de Chile, Naciones Unidas.

CEPAL. (2016). La nueva revolución digital. De la internet del consumo a la internet de la producción. Santiago de Chile, Naciones Unidas.

CHAN, Anita. (2004). Coding Free Software, Coding Free States: Free Software Legislation and the Politics of Code in Peru. Anthropological Quarterly, 77, 3: 531-545.

COLIBRÍ. (2014, Junio 24). Hagas lo que hagas juega limpio usa software libre. Recuperado de http://www.mail-archive.com/colibri@listas. el-directorio.org/msg15068.html

COLIBRÍ. (2011, Marzo 24). Ley sobre Software Libre en Colombia. Recuperado de http://colibri. listas.el-directorio.narkive.com/RhcvfeTc/leysobre-software-libre-en-colombia

COLIBRÍ. (2009, Octubre 19). Proyecto Ley Software Libre. Recuperado de http://software-librecolombia.2324882.n4.nabble.com/ProyectoLey-Software-Libre-td1 1272.html

COLIBRÍ. (2007, Noviembre 13). Advertencia sobre neutralidad. Recuperado de http://www. mail-archive.com/colibri@listas.el-directorio. org/msg02743.html

COLIBRÍ. (2007, Septiembre 26). Audiencia pública proyecto de Ley software libre. Recuperado de http://www.mail-archive.com/colibri@listas. el-directorio.org/msg02319.html

CONGRESO DE LA REPÚBLICA. (2010). Ponencia para primer debate al Proyecto de Ley 017 de 2009. Gaceta del Congreso, 432.

CONGRESO DE LA REPÚBLICA. (2009). Proyecto de Ley 017 de 2009. Gaceta del Congreso, 603.

CONGRESO DE LA REPÚBLICA. (2008). Ponencia para segundo debate al Proyecto de Ley 021 de 2007. Gaceta del Congreso, 45.

CONGRESO DE LA REPÚBLICA. (2007). Proyecto de Ley 021 de 2007. Gaceta del Congreso, 339.

CONGRESO DE LA REPÚBLICA. (2003). Ponencia para primer debate al Proyecto de Ley 83 de 2002. Gaceta del Congreso, 228.

CONGRESO DE LA REPÚBLICA. (2002). Proyecto de ley 83 de 2002. Gaceta del Congreso, 402.

CONTRERAS, José. (2013). Resistencia y Libertad. Parte II. La revolución chavista y la revolución del software libre se encuentran. ¿Se encuentran? En: ROCA, Santiago. (Compilador). Reflexiones sobre el impacto transformador de las tecnologías libres. A propósito del Infogobierno en Venezuela. Caracas, Fundación Centro Nacional de Desarrollo e Investigación en Tecnologías Libres. pp. 135-154.

CORTÉS, Carlos. (2004). Microsoft entre monopolio y ciberseguridad. Chasqui. Revista Latinoamericana de Comunicación, 85: 72-79.

CROWSTON, Kevin; LI, Qing; WEI, Kangning; ESERYEL, Yeliz \& HOWISON, James. (2007). Self-organization of teams for free/libre open source software deevlopment. Information and Software Technology, 49, 6: 564-575.

D’ELIA, Marcelo; LEÓN, Mónica; NOVO, Alejandro \& OTERO, Alberto. (2005). Software Libre. Implantación de sistemas. Barcelona, Fundació per a la Universitat Oberta de Catalunya.

DE GREIFF, Alexis. (2017). Infraestructura y distribución social de objetos digitales culturales. En: GÖBEL, Bárbara \& CHICOTE, Gloria. (Editoras). Transiciones inciertas. Archivos, conocimientos y transformación digital en América Latina. La 
Plata, Facultad de Humanidades y Ciencias de la Educación; Berlín, Ibero-Amerikanisches Institut, pp. 80-97

DE LAAT, Paul. (2005). Copyright or Copyleft?: An analysis of property regimes for software development. Research Policy, 34, 10: 1511-1532.

DELGADO, Ana María \& CUELLO, Rafael. (2007). La promoción del uso del software libre por parte de las universidades. Revista de Educación a Distancia, 17. Recuperado de http://revistas. um.es/red/article/view/24221

DOLCEMÁSCOLO, Agostina. (2013). Aplicación del software libre en la administración pública: una reflexión sobre el caso de Brasil. Question, 1, 37: 71-82.

ESTEPA, Jesús. (2007). Software Libre para el Desarrollo del Tercer Mundo. (Proyecto de Grado). Granada, Universidad de Granada.

EVANGELISTA, Rafael. (2014). O movimiento software livre do Brasil. Política, trabalho e hacking. Antropologia e Politicas Globais, 41: 173-200.

FRANKLIN, M.I. (2013). Digital Dilemmas. Power, Resistance and the Internet. New York: Oxford University Press.

GÓMEZ, Rafael. (2003). Software Libre vs. Software Propietario: Programando Nuestro Futuro. Historia Actual Online, 2: 125-140.

GONZÁLEZ, Jesús. (2003). El futuro de la información: ¿vamos hacia donde queremos? Archipiélago, 55.

GUESSER, Adalto. (2006). Software Livre e Controvérsias Tecnocientificas: Uma Análise Sociotécnica no Brasil e em Portugal. Curitiba, Juruá Editora.

HESS, David. (2005). Technology -and ProductsOriented Movements: Approximating Social Movements Studies and Science and Technology Studies. Science, Technology and Human Values, 30, 4: 515-535.

HIMANEN, Pekka. (2001). La ética del hacker y el espíritu de la era de la información. Recuperado de http://eprints.rclis.org/12851/1/pekka.pdf

HINE, Christine. (2004). Etnografía virtual. Barcelona, Editorial UOC.

KELLNER, Douglas. (2001). Globalisation, Technopolitics and Revolution. Theoria: $A$ Journal of Social and Political Theory, 98: 14-34.
KELTY, Christopher. (2004). Culture's Open Source: Software, Copyright and Cultural Critique. Antropological Quarterly, 77, 3: 499-506.

KIM, Hongbum; SHIN, Dong-Hee \& LEE, Daeho. (2015). A socio-technical analysis of software policy in Korea: Towards a central role for building ICT ecosystems. Telecommunications Policy, 39: 944-956.

LATOUR, Bruno. (2010). The Making of Law. An Ethnography of the Conseil D 'Etat. Cambridge, Polity Press.

LATOUR, Bruno. (1992). Ciencia en acción. Cómo seguir a los cientificos e ingenieros a través de la sociedad. Barcelona, Editorial Labor.

LATOUR, B. \& WOOLGAR, Steve. (1995). La vida en el laboratorio. La construcción de los hechos cientificos. Madrid, Alianza Universidad.

LESSIG, Lawrence. (2005). Por una cultura libre. Barcelona, Traficante de sueños.

MARTÍNEZ, Patricia. (2018). "Seguro mató a confianza": desafíos para la adopción del gobierno digital en Colombia. Inclusión y Desarrollo, 5, 1: 63-72.

MEISZNER, Andreas; GLOTT, Rüdiger \& SOWE, Sulayman. (2008). Free/Libre Open Source Software (FLOSS) Communities as an Example of Succesful Open Participatory Learning Ecosystems. The European Journal for the Informatics Professional, 9, 3: 62-68.

MINISTERIO DE LAS TIC. (2015). Computadores para educar: caso de éxito para Microsoft. Recuperado de https://bit.ly/32DUzfi

MIRA. (2009). Proyectos de Ley presentados por el partido MIRA. Recuperado de https://partidomira. $\mathrm{com} /$ ?s=proyectos + de + ley

NYE, David. (2006). Technology Matters: Questions to Live With. Cambridge, MIT Press.

PÉREZ-BUSTOS, Tania. (2010). Reflexiones sobre una etnografía feminista del Software Libre en Colombia. Revista Estudos Feministas, 18, 2: 385-405.

RAYMOND, Eric. (1999). The Catedral and the Bazar. Musings on Linux and Open Source by an Accidental Revolutionary. California, O’Reilly Media Inc. 
REINA, Daniel. (2006). Criterios a migración Linux en las administraciones locales de la UE. Los casos de Munich y Newham. Uoc Papers Revista sobre la sociedad del conocimiento, 2: 1-8.

ROBERTS, Ben. (2011). Against the "Networked Information Economy": Rethinking Decentralization, Community, and Free Software Development. Criticism, 53, 3: 385-405.

ROCA, Santiago \& MONTILLA, Marcela. (2013). Significado de las Tecnologías de Información Libres para el Futuro de la Sociedad Venezolana. En: ROCA, Santiago. (Compilador). Reflexiones sobre el impacto transformador de las tecnologias libres. A propósito del Infogobierno en Venezuela. Caracas, Fundación Centro Nacional de Desarrollo e Investigación en Tecnologías Libres. pp. 111-122.

RUIZ, Miguel. (2008). Ciberetnografía: comunidad y territorio en el entorno virtual. En: ARDĖVOL, Elisenda; ESTATELLA, Adolfo \& DOMÍNGUEZ, Daniel. (Coords). La mediación tecnológica en la práctica etnográfica. Donostia, Ankulegi Antropología Elkartea: 117-132.

SABATER, Carmen; MARTÍNEZ, Ion \& SANTIAGO, Raúl. (2017). La Tecnosociedad. El papel de las TIC en las relaciones sociales. Revista Latina de Comunicación Social, 72, 14: 1592-1607.

SANTOS, Leonardo. (2013). Cultura do software livre e desenvolvimento: Uma análise sobre potencialidades e limites diante e adiante da "nova economía". Revista Crítica de Ciencias Sociais, 102: 71-88.
SCARSO, Enrico. (2008). The Role of Knowledge Mediators in Virtual Environments. En BOLISARI, Ettore. (Editor). Building the Knowkedge Society on the Internet. Sharing and Exchanging Knowledge in Networked Environments. New York, Information Science Reference, pp. 282-301.

SÖDERBERG, Johan. (2008). Hacking Capitalism. The Free and Open Source Software Movement. New York, Routledge.

STALLMAN, Richard. (2014). El software libre es un asunto ético del uso de la tecnología. Conferencia dictada en el Auditorio Gorbea de la Facultad de Ciencias y Matemáticas de la Universidad de Chile. Recuperado de https://www.dcc.uchile. cl/richard_stallman

STALLMAN, Richard. (2004). Software libre para una sociedad libre. Madrid, Traficantes de sueños.

VERA, Paula. (2013). Imaginarios tecnológicos y procesos de construcción urbana en la ciudad moderna. El ferrocarril, el automóvil y las TIC. Revista de Estudios Urbanos y Ciencias Sociales, 3 (1): 9-26.

WHITE, Lynn. (1990). Tecnología medieval y cambio social. Barcelona, Paidós.

WINOCUR, Rosalía. (2007). La apropiación de las TIC en la vida cotidiana. Telos, 73: 109-117.

WINNER, Langdon. (1992). The Whale and the Reactor. A Search for Limits in a Age of High Technology. Chicago: The University od Chicago Press. 


\section{LOS PROYECTOS DE LEY SOBRE SOFTWARE LIBRE EN COLOMBIA \\ OS PROJETOS DE LEI SOBRE SOFTWARE LIVRE NA COLÔMBIA}

\section{Alexander Pereira-García}

Palabras clave: Software Libre; Legislación Tecnológica; Etnografía Virtual; Regular el software.

El presente artículo es un análisis sobre los proyectos de ley presentados ante el Congreso de la República con la intención de legislar el software libre en Colombia, los cuales no fueron exitosos en su trámite legislativo. Se analizan los tránsitos generados en las propuestas legislativas y las razones por las cuales estas no fueron aprobadas, haciendo énfasis en la postura de los grupos defensores del software libre. Con este fin se propuso la revisión de documentos legislativos publicados en los medios oficiales colombianos, además del abordaje a diversos actores involucrados en las controversias desde la perspectiva de la etnografía virtual, en la cual se abordan los espacios virtuales, como mecanismo para entender las dinámicas que ocurrieron entre diversos actores involucrados en el debate. Se afirma que el fracaso de los proyectos de ley sobre software libre en Colombia evidencia los intereses de grupos dominantes que establecen alianzas influyentes en el curso de la regulación tecnológica, además, de la alineación del discurso tecnológico con intereses mercantiles.

\section{Alexander Pereira-García}

Palavras-chave: Software Livre; Legislação Tecnológica; Etnografia Virtual; Regulamentação do software.

O presente artigo abrange uma análise sobre os projetos de lei apresentados ante o Congresso da República da Colômbia como esforços para regulamentar o software livre no país, os quais náo foram exitosos no seu trâmite legislativo. Analisam se os trânsitos gerados nas propostas legislativas e as razóes pelas quais não foram aprovadas, fazendo ênfases na postura dos grupos defensores do software livre. Portanto, se propôs a revisão de documentos legislativos publicados nas mídias oficiais colombianas, levando em conta a abordagem de diversos atores involucrados nas controvérsias desde a perspectiva da etnografia virtual, na qual se abrangem os espaços virtuais como mecanismo para entender as dinâmicas que aconteceram entre os atores involucrados no debate. No final, afirma-se que o fracasso dos projetos de lei sobre software livre na Colômbia evidencia os interesses de grupos dominantes que estabelecem alianças influentes no curso da regulação tecnológica, além disso, de um discurso tecnológico com interesses mercantis.

\section{FREE SOFTWARE LAW PROJECTS IN COLOMBIA}

\section{Alexander Pereira-García}

Keywords: Free Software; Technological Legislation; Virtual Ethnography; Software Regulation.

This article covers an analysis of the bills presented before the Republic of Colombia Congress as efforts to regulate free software in the country, which were not successful in their legislative process. It analyzes whether the transits generated in the legislative proposals and the reasons why they were not approved, emphasizing the position of groups that defend free software. Therefore, a revision of legislative documents published in the Colombian official media was proposed, taking into account the approach of several actors involved in controversies from a virtual ethnography perspective, in which virtual spaces are included as a mechanism to understand the dynamics between the actors involved in the debate. In the end, it is affirmed that the failure of free software bills in Colombia highlights the interests of dominant groups that establish influential alliances in the course of technological regulation, in addition to a technological discourse with market interests. 


\section{LES PROJETS DE LOI SUR LE LOGICIEL LIBRE EN COLOMBIE}

\section{Alexander Pereira-García}

Mots-clés: Logiciels libres; législation technologique; ethnographie virtuelle; réglementation des logiciels.

L'article analyse l'échec des projets de lois soumis au Congrès de la république de Colombie sur la réglementation du logiciel libre dans le pays. Il examine le parcours des propositions législatives et les raisons de leur non-approbation tout en mettant l'accent sur la position des partisans du logiciel libre. L'objectif est d'étudier les documents législatifs publiés dans la presse officielle colombienne en tenant compte de l'approche de différents acteurs impliqués dans les controverses depuis la perspective de l'ethnographie virtuelle, où les espaces virtuels sont un mécanisme qui permet de comprendre les dynamiques entre les participants du débat. La recherche montre que l'échec des projets de loi sur le logiciel libre en Colombie est lié aux intérêts de groupes dominants qui établissent des alliances influentes au niveau de la régulation technologique et dont le discours technologique est dominé par des intérêts commerciaux. 